\title{
Fecal microbiota transplantation for refractory Crohn's disease
}

\author{
Seon Ho Bak ${ }^{1}$, Hyun Ho Choi ${ }^{2}$, Jinhee Lee ${ }^{1}$, Mi Hee Kim ${ }^{1}$ Youn Hee Lee ${ }^{1}$, Jin Su Kim ${ }^{1}$, Young-Seok Cho ${ }^{1}$ \\ ${ }^{1}$ Department of Internal Medicine, Seoul St. Mary's Hospital, College of Medicine, The Catholic University of Korea, Seoul, \\ ${ }^{2}$ Department of Internal Medicine, Uijeongbu St. Mary's Hospital, College of Medicine, The Catholic University of Korea, Uijeongbu, Korea
}

Approximately one-third of patients with Crohn's disease do not respond to conventional treatments, and some experience significant adverse effects, such as serious infections and lymphoma, and many patients require surgery due to complications. Increasing evidence suggests that specific changes in the composition of gut microbiota, termed as dysbiosis, are a common feature in patients with inflammatory bowel disease (IBD). Dysbiosis can lead to activation of the mucosal immune system, resulting in chronic inflammation and the development of mucosal lesions. Recently, fecal microbiota transplantation, aimed at modifying the composition of gut microbiota to overcome dysbiosis, has become a potential alternative therapeutic option for IBD. Herein, we present a patient with Crohn's colitis in whom biologic therapy failed previously, but clinical remission and endoscopic improvement was achieved after a single fecal microbiota transplantation infusion. (Intest Res 2017;15:244-248)

Key Words: Crohn disease; Fecal microbiota transplantation; Dysbiosis; Biologic therapy

\section{INTRODUCTION}

Crohn's disease (CD) is a chronic relapsing intestinal inflammatory disorder. It is characterized by progressive transmural inflammation of the gastrointestinal tract, which often leads to structuring or penetrating complications requiring surgical resection. ${ }^{1}$ Although the etiology of the disease remains unclear, CD involves interactions among genetic susceptibility, environmental factors (antigens derived from commensal bacteria), intestinal microbiota, and the immune system. ${ }^{2}$ Over the past decades, the incidence and prevalence of CD have gradually increased in Asian countries, including Korea. ${ }^{.}$Conventional treatment for $\mathrm{CD}$ is based on the administration of aminosalicylates, corticosteroids, and

Received August 23, 2015. Revised December 10, 2015. Accepted December 21, 2015. Published online March 3, 2017

Correspondence to Young-Seok Cho, Department of Internal Medicine, Seoul St. Mary's Hospital, College of Medicine, The Catholic University of Korea, 222 Banpo-daero, Seocho-gu, Seoul 06591, Korea. Tel: +82-2-22586021, Fax: +82-2-2258-2038,E-mail: yscho@catholic.ac.kr

Financial support: None. Conflict of interest: None. immunosuppressants prior to escalation to biological therapies, in particular those using tumor necrosis factor- $\alpha$ antibodies. ${ }^{3}$ However, approximately one-third of patients do not respond to these treatments, some experience significant adverse effects, such as serious infections and lymphoma, and many patients require surgery. ${ }^{4}$

Increasing evidence suggests that specific changes in the composition of gut microbiota, termed dysbiosis, are a common feature in patients with IBD, including UC and CD. ${ }^{2}$ Dysbiosis can lead to activation of the mucosal immune system, resulting in chronic inflammation and the development of mucosal lesions. Recently, fecal microbiota transplantation (FMT), aimed at modifying the composition of gut microbiota to overcome dysbiosis, has become a potential alternative therapeutic option for IBD. ${ }^{5,6}$ Herein, we present a patient with $\mathrm{CD}$ in whom biological therapy failed previously, but clinical remission and endoscopic improvement was achieved after a single FMT infusion.

\footnotetext{
(c) Copyright 2017. Korean Association for the Study of Intestinal Diseases. All rights reserved.

This is an Open Access article distributed under the terms of the Creative Commons Attribution Non-Commercial License (http://creativecommons.org/licenses/by-nc/4.0)

which permits unrestricted non-commercial use, distribution, and reproduction in any medium, provided the original work is properly cited.
} 


\section{CASE REPORT}

A 16-year-old female patient was admitted to Uijeongbu St. Mary's Hospital for the management of refractory colonic CD (A1L2B1 Montreal classification for Crohn's disease 2005). CD was diagnosed 1 year prior, according to standard endoscopic and histologic criteria (Fig. 1). CD was remitted with prednisone, and maintenance therapy with mesalazine and 6-mercaptoprine was administered. Four months prior to hospital admission, she was treated with infliximab (5 mg/ $\mathrm{kg}$ ) because of several flares, and she responded well; however, colitis aggravated 1 month before admission. On admission, the patient's body temperature was $37.1^{\circ} \mathrm{C}$, and she had poor general condition. Laboratory examination showed the following: leukocyte count, $8,260 / \mathrm{mm}^{3}$ with $51.7 \%$ neutrophils; hemoglobin, $8.1 \mathrm{~g} / \mathrm{dL}$; ESR, $36 \mathrm{~mm} / \mathrm{h}$; CRP level, 6.17 $\mathrm{mg} / \mathrm{dL}$; and serum albumin level, $3.0 \mathrm{~g} / \mathrm{dL}$. A stool specimen contained white blood cells, and a test for Clostridium difficile toxin was negative. Immunohistochemical staining for cytomegalovirus was negative. Even after the infliximab dose was increased to $10 \mathrm{mg} / \mathrm{kg}$, repeat colonoscopy showed active colitis (Fig. 2), and the patient became steroid dependent.

Her CDAI score was 394; thus, treatment with FMT was discussed with the patient and her parents, and they agreed to treatment. The FMT procedure was performed on day 12 after hospital admission. The FMT stool source, a family donor, received both blood and stool tests, and HBsAg, antiHCV, Veneral Disease Research Laboratory test, $C$. difficile toxin, and human immunodeficiency virus were not detect-

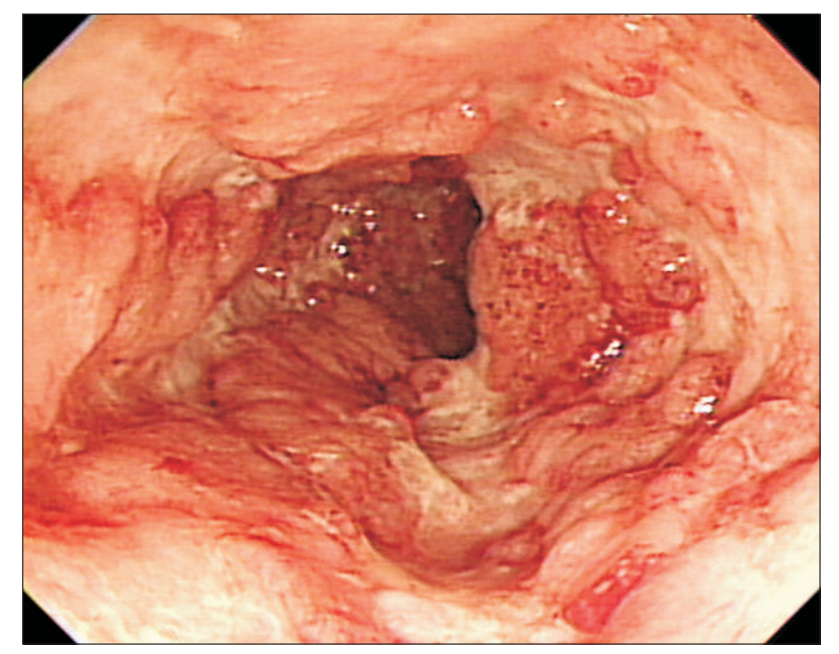

Fig. 1. Initial colonoscopic finding. Colonoscopy at the time of diagnosis reveals deep longitudinal ulcerations in the descending colon. ed. The donor had no history of antibiotic use within the past year or any history of chemotherapy. Sixty grams of stool was mixed in $250 \mathrm{~mL}$ of normal saline and homogenized using a mechanical blender. The suspension was filtered through gauze to remove larger particulate matters. FMT was performed using gastroduodenoscopy; the endoscope was inserted into the second portion of the duodenum, and the prepared fecal suspension was transferred to the patient's bowels through the biopsy channel of the endoscope. The procedure time was approximately 5 minutes. One day after FMT, the patient developed abdominal pain and a transient fever; however, these symptoms disappeared after conservative care. One week after FMT, the patient felt well

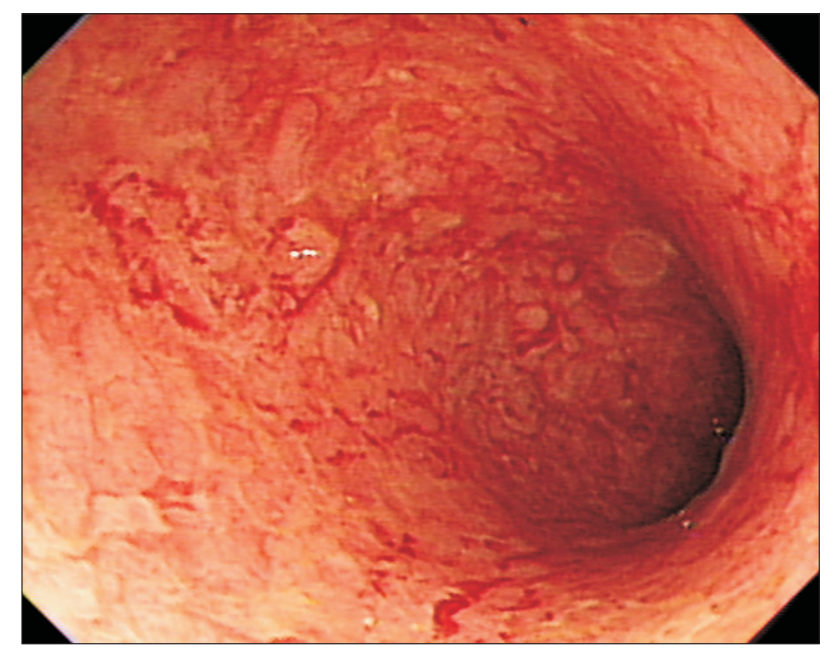

Fig. 2. Repeat colonoscopic finding. Colonoscopy shows active colitis in the sigmoid colon even after biological therapy was administered.

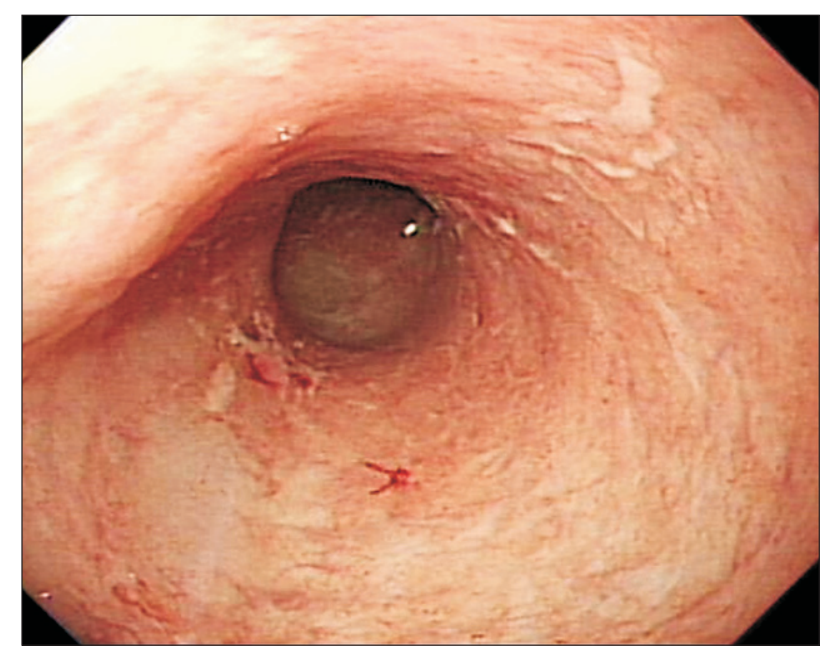

Fig. 3. Colonoscopic finding after fecal microbiota transplantation (FMT). Follow-up colonoscopy 10 months after FMT shows improvement in mucosal lesions. 
and had 1 to 2 formed bowel movements per day with no abdominal pain. The CDAI was reduced to 132 , and the patient was discharged. Although clinical remission was achieved, the patient wished to receive maintenance treatment. After FMT, mesalazine ( $3.0 \mathrm{~g} /$ day) and azathioprine $(50 \mathrm{mg} /$ day) was administered for maintenance therapy. In addition, 2 months after FMT, she was administered infliximab (5 mg/kg) intravenously, to which she responded well, and treatment with infliximab was continued every 2 months. A follow-up colonoscopy 10 months after FMT showed improvement in the mucosal lesions (Fig. 3). Clinical remission was sustained for more than 12 months, and the follow-up is ongoing (Fig. 4).
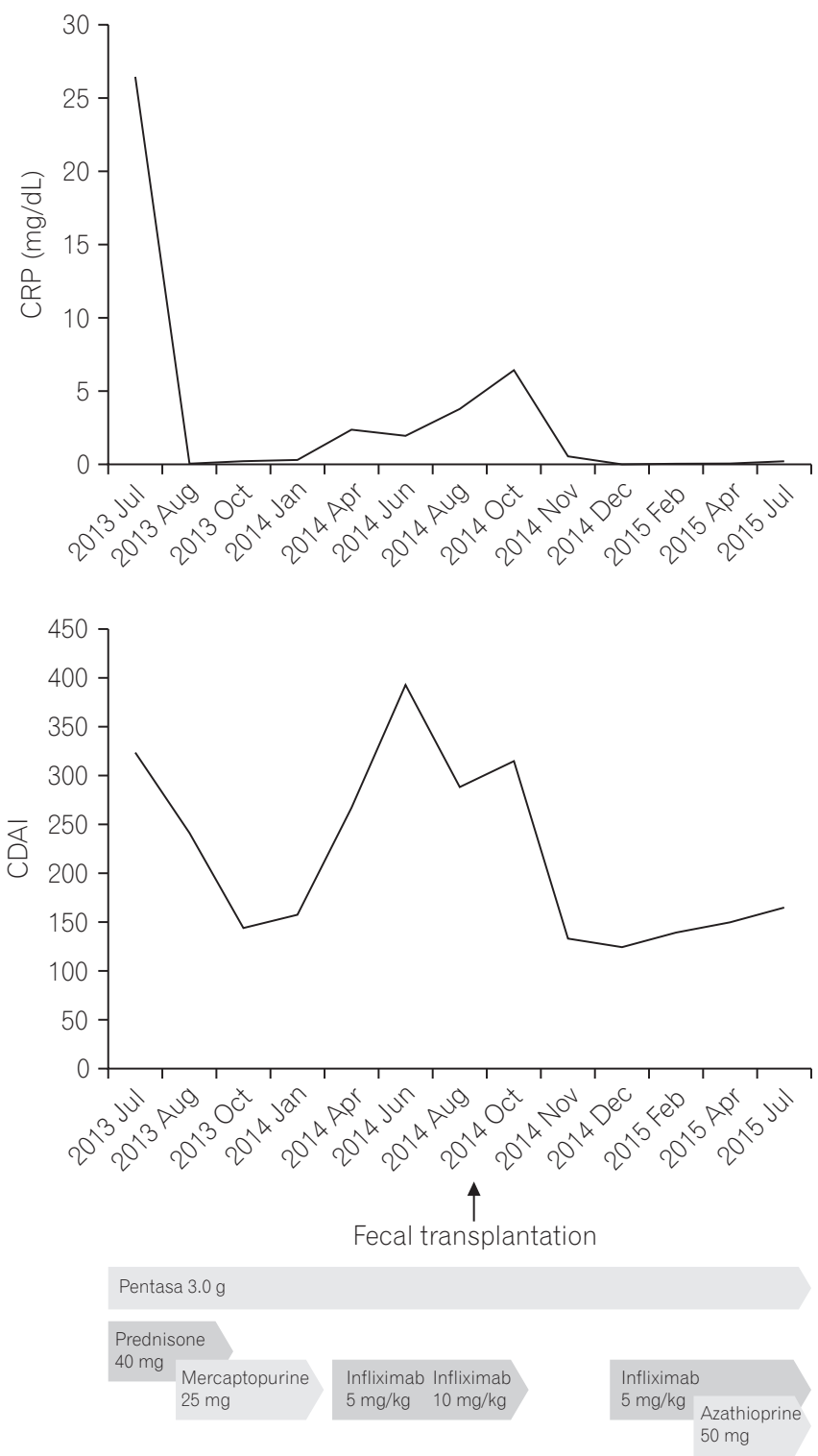

Fig. 4. Clinical course of CRP and CDAl during medical therapy.

\section{DISCUSSION}

FMT, also referred to as stool/fecal transplantation or fecal bacteriotherapy, is the infusion or engraftment of fecal liquid filtrate from a healthy donor into the gut of a recipient to cure a specific disease. ${ }^{6}$ The concept of FMT for treatment of human intestinal diseases was described in China during 4 th century, and human fecal suspension by mouth was used to treat patients who had food poisoning or severe diarrhea. Although it was first reported in $1958,{ }^{8}$ it has only recently become popular due to its success in treating refractory and recurrent $C$. difficile infections (CDI). Recent studies have shown that FMT is an effective treatment in recurrent CDI, with a $>90 \%$ success rate, and it can be considered as an antibiotic replacement for recurrent and refractory CDI that has relapsed more than three times. ${ }^{9}$

Although no definitive pathogen has been detected as the etiologic factor of IBD, many studies have investigated the disturbance of intestinal microbiota as a contributing factor to IBD pathogenesis. ${ }^{4}$ Consistent alterations in intestinal microbiota in IBD are characterized by decreased diversity at the species level, with notable decreases in the Bacteroides phylum and Lachnospiraceae group within the Firmicutes phylum and increases in Proteobacteria and Actinobacteria. ${ }^{6}$ In addition, a decrease in a butyrate-producing bacterium, which is important in intestinal health, has been observed in patients with IBD. ${ }^{10}$ However, it is still unclear whether dysbiosis itself causes IBD or if it represents an epiphenomenon due to microbial alterations as a consequence of the disease. $^{4}$

The application of FMT for IBD was first published in 1989 as a case report, in which the author himself had confirmed UC for 7 years that was refractory to both sulfasalazine and steroids. Six months after transplantation of a healthy donor stool by retention enema, he remained symptom-free, and active inflammation was not detected during a follow-up biopsy sampling of the colon. ${ }^{11}$ Initial case studies of FMT enemas in patients with IBD reported that many of the patients achieved clinical remission and maintained long-term remission, and a small number of these cases also reported endoscopic and histological remission. ${ }^{12}$ However, subsequent small case series of FMT in children and adults with UC, CD, or pouchitis showed mixed results. ${ }^{6}$ Despite the demonstrated safety and efficacy of FMT for IBD in these case studies, the available evidence is scarce and insufficient as these studies were underpowered and open-labeled, and a lack of uniformity exists among FMT protocols and defined outcomes. ${ }^{6}$ Moreover, the patient populations were 
heterogeneous in disease type and severity, phenotype, and concomitant medications.

A recent systematic review and meta-analysis of 18 studies (nine cohort studies, eight case studies, and one randomized controlled trial) on FMT that included 122 patients with IBD (79 UC, 39 CD, and four unclassified) demonstrated a clinical remission rate of $45 \%{ }^{5}$ However, the pooled estimate for achieving short-term clinical remission after FMT was $36.2 \%$, after excluding case studies to minimize publication bias. Subgroup analyses showed a pooled estimate of clinical remission of $22 \%$ (95\% CI, 10.4-40.8) for UC and $60.5 \%$ (95\% CI, 28.4-85.6) for CD. In addition, the pooled estimate for clinical remission was highest $(64.1 \%)$ in young patients (aged, 7-20 years). However, the high pooled estimates for clinical remission in patients with $\mathrm{CD}$ or in young patients were significantly heterogeneous, which considerably limits the applicability of these conclusions. Cui et al. ${ }^{13}$ recently published a pilot study in which thirty patients with refractory CD were treated with a single FMT through the mid-gut. The rates of clinical improvement and remission based on clinical activity during the first month after FMT were 86.7\% $(26 / 30)$ and $76.7 \%$ (23/30), respectively. In addition, the body weights of the patients increased.

Recently, the first two randomized placebo-controlled trials evaluating the efficacy of FMT for UC were reported. In one study, 75 patients with mild-to-moderate UC were randomized to weekly FMT or placebo (water) via retention enema for 6 weeks, and the patients who received FMT had a significantly greater remission rate than that of those receiving the placebo ( $24 \%$ vs. $5 \%$ ). ${ }^{14}$ In addition, a microbiomic analysis showed that patients who received FMT had greater microbial diversity than those who received the placebo. The other study enrolled 50 patients with mild-to-moderately active UC and randomized them to either donor stool or autologous FMT delivered via a nasoduodenal tube at baseline and again 3 weeks later. ${ }^{15}$ Only 37 patients completed the assessment for primary endpoint-clinical remission combined with $a \geq 1$-point decrease in the Mayo endoscopic score at week 12. There were no differences in clinical or endoscopic remission between the two groups. However, patients who responded favorably showed a microbiota profile similar to that of their respective donors, whereas nonresponders did not show the same trend. Both studies were discontinued based on their respective data and safety monitoring boards due to futility in reaching their primary efficacy points.

Although several cases of IBD treated by FMT have been reported worldwide, there are currently no such cases in Korea. To our knowledge, the current article is the first report of such a case in Korea. FMT via the lower gastrointestinal route is preferred, but colonoscopy may be potentially dangerous in a patient with severe colitis. ${ }^{4}$ Therefore, our patient was administered fecal suspension via the upper gastrointestinal route. Although fecal microbial analysis was not performed before or after FMT in our patient, it is an interesting direction for future research. Taken together, FMT is not as effective for IBD treatment as it is for CDI, suggesting that IBD is a more complicated and heterogeneous disease with complex interactions among genetic, environmental, immunologic, and gut microbial factors.

Although previous studies reported that patients with IBD undergoing FMT need to receive antibiotics, further study is required to elucidate whether antibiotic treatment before FMT is necessary. ${ }^{4}$ Our patient was not administered antibiotics as a pretreatment. Overall, FMT is considered relatively tolerable and safe in the short term. Commonly reported immediate adverse events include abdominal discomfort, bloating, flatulence, diarrhea, constipation, vomiting, and transient fever. ${ }^{6}$ Most of these symptoms are self-limiting and disappear within 2 days after the FMT procedure. ${ }^{4}$ However, very little information is available regarding the long-term immunologic effects of FMT, including the onset of latent infections. In addition, diseases or conditions related to changes in gut microbiota may occur, including obesity, diabetes, atherosclerosis, IBD, colon cancer, nonalcoholic fatty liver disease, IBS, asthma, and autism. ${ }^{6}$ Long-term follow-up data for FMT should be investigated further.

In conclusion, this case report suggests that FMT through the mid-gut may be an optimal treatment for refractory CD unresponsive to current conventional therapy, such as antiinflammatory agents, steroids, immunosuppressives, and biological therapies. Further prospective randomized studies are necessary to fully assess the safety and efficacy of FMT in patients with IBD.

\section{REFERENCES}

1. Lee KM, Lee JM. Crohn's disease in Korea: past, present, and future. Korean J Intern Med 2014;29:558-570.

2. Manichanh C, Borruel N, Casellas F, Guarner F. The gut microbiota in IBD. Nat Rev Gastroenterol Hepatol 2012;9:599-608.

3. Kim NH, Jung YS, Moon CM, et al. Long-term clinical outcomes of Korean patient with Crohn's disease following early use of infliximab. Intest Res 2014;12:281-286. 
4. Wang ZK, Yang YS, Chen Y, Yuan J, Sun G, Peng LH. Intestinal microbiota pathogenesis and fecal microbiota transplantation for inflammatory bowel disease. World J Gastroenterol 2014;20:14805-14820.

5. Colman RJ, Rubin DT. Fecal microbiota transplantation as therapy for inflammatory bowel disease: a systematic review and meta-analysis. J Crohns Colitis 2014;8:1569-1581.

6. Kelly CR, Kahn S, Kashyap P, et al. Update on fecal microbiota transplantation 2015: indications, methodologies, mechanisms, and outlook. Gastroenterology 2015;149:223-237.

7. Zhang F, Luo W, Shi Y, Fan Z, Ji G. Should we standardize the 1,700-year-old fecal microbiota transplantation? Am J Gastroenterol 2012;107:1755.

8. Eiseman B, Silen W, Bascom GS, Kauvar AJ. Fecal enema as an adjunct in the treatment of pseudomembranous enterocolitis. Surgery 1958;44:854-859.

9. Drekonja D, Reich J, Gezahegn S, et al. Fecal microbiota transplantation for Clostridium difficile infection: a systematic review. Ann Intern Med 2015;162:630-638.
10. Cammarota G, Ianiro G, Cianci R, Bibbò S, Gasbarrini A, Currò D. The involvement of gut microbiota in inflammatory bowel disease pathogenesis: potential for therapy. Pharmacol Ther 2015;149:191-212.

11. Bennet JD, Brinkman M. Treatment of ulcerative colitis by implantation of normal colonic flora. Lancet 1989;1:164.

12. Brandt LJ, Aroniadis OC. An overview of fecal microbiota transplantation: techniques, indications, and outcomes. Gastrointest Endosc 2013;78:240-249.

13. Cui B, Feng Q, Wang H, et al. Fecal microbiota transplantation through mid-gut for refractory Crohn's disease: safety, feasibility, and efficacy trial results. J Gastroenterol Hepatol 2015;30:5158.

14. Moayyedi P, Surette MG, Kim PT, et al. Fecal microbiota transplantation induces remission in patients with active ulcerative colitis in a randomized controlled trial. Gastroenterology 2015;149:102-109.e6.

15. Rossen NG, Fuentes S, van der Spek MJ, et al. findings from a randomized controlled trial of fecal transplantation for patients with ulcerative colitis. Gastroenterology 2015;149:110-118.e4. 